\title{
User-centered EEG-based Multimedia Quality Assessment
}

\author{
Arghir-Nicolae Moldovan, Ioana Ghergulescu, Stephan Weibelzahl and Cristina Hava Muntean
}

\begin{abstract}
Multimedia users are becoming increasingly quality-aware as the technological advances make ubiquitous the creation and delivery of high-definition multimedia content. While much research work has been conducted on multimedia quality assessment, most of the existing solutions come with their own limitations, with particular solutions being more suitable to assess particular aspects related to user's Quality of Experience (QoE). In this context, there is an increasing need for innovative solutions to assess user's QoE with multimedia services. This paper proposes the QoE-EEG-Analyser that provides a solution to automatically assess and quantify the impact of various factors contributing to user's QoE with multimedia services. The proposed approach makes use of participant's frustration level measured with a consumer-grade EEG system, the Emotiv EPOC. The main advantage of QoE-EEG-Analyser is that it enables continuous assessment of various $\mathrm{QoE}$ factors over the entire testing duration, in a non-invasive way, without requiring the user to provide input about his perceived visual quality. Preliminary subjective results have shown that frustration can indicate user's perceived QoE.
\end{abstract}

Index Terms-Quality of Experience, multimedia quality assessment, subjective assessment methods, objective quality metrics, EEG.

\section{INTRODUCTION}

$\mathrm{M}$ ULTIMEDIA services such as video sharing, video-ondemand, IPTV, and video conferencing are increasing fast in popularity, with the network traffic generated by these services being projected to increase three times between 2012 and 2016 , and to reach $55 \%$ of the global Internet traffic by 2016 [1]. At the same time, users increasingly use multiple devices such as smartphones, tablets, PCs and even smart TVs for conducting online activities in general and for accessing multimedia services in particular [2]. With the proliferation of high-resolution device displays and powerful video cameras integrated with mobile devices, of high-definition multimedia content, as well as of high-speed wireless networks that enable delivering this content, users' expectations in terms of the quality offered by multimedia services are also increasing.

There are many factors contributing to user's perceived quality of multimedia services, with research and industry alike, gradually shifting from the notion of Quality of Service

Manuscript received May 9, 2013. This work was supported by Irish Research Council's EMBARK Initiative.

A.-N. Moldovan, I. Ghergulescu, S. Weibelzahl and C. H. Muntean, are with the School of Computing, National College of Ireland, Mayor Street, IFSC, Dublin 1, Ireland (e-mail: amoldovan@student.ncirl.ie; ioana.ghergulescu@student.ncirl.ie; sweibelzahl@ncirl.ie; cmuntean@ncirl.ie).

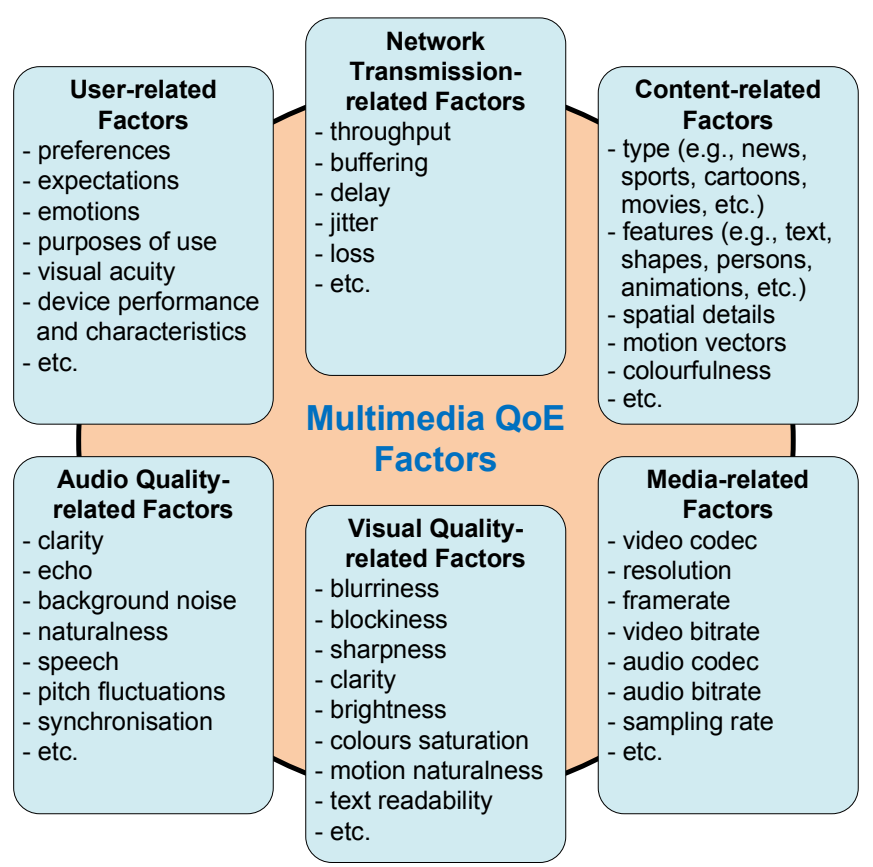

Fig.1. Factors contributing to user's QoE with multimedia services.

(QoS), to broader aspects such as Quality of Perception (QoP) or Quality of Experience (QoE) [3],[4], [5]. Figure 1 presents a list of multimedia QoE factors, grouped in different categories such as: user-related, network transmission-related, content-related, media-related, visual quality-related and audio quality-related factors. This list is not an exhaustive one and many other factors can contribute as well to a user's QoE.

The high number of factors and combinations in which they may occur, makes it very difficult to assess user's QoE. Although many solutions for multimedia quality assessment have been proposed, this area is far from being fully understood and continues to draw much attention from researchers, industry and standardisation organizations.

Subjective assessment consisting of a number of participants providing input (e.g., perceived multimedia quality ratings) relative to their $\mathrm{QoE}$ with the multimedia service, is usually considered the most accurate and reliable. However, this approach presents significant limitations when it comes to assess and quantify the contribution of multiple QoE factors as participants would be disturbed from their natural viewing experience due to the interaction taking place during the subjective study. At the same time, existing objective metrics that aim to enable automatic multimedia 
quality assessment without user input, usually tend to offer mixed performance as they exploit various content characteristics and artifacts (e.g., blurriness, blockiness, etc.), or have requirements such as for example strict temporalspatial synchronization that render them unsuitable in case of adapted streams with varying characteristics (e.g., resolution, frame rate, etc.), and/or network transmission-related distortions (e.g. delays, frames loss, etc.) [6].

In order to overcome many of the challenges with multimedia delivery such as the diversity of device characteristics and the variable network conditions, adaptive multimedia streaming has evolved from a mainly research topic few years ago to a number of industry solutions and standards being available nowadays [7].

In this context, this paper aims to propose a hybrid subjective-objective solution for automatically assessing and quantifying the impact of multiple factors contributing to user's QoE with adaptive multimedia services. Instead of asking the participants to provide input, the proposed QoEEEG-Analyser uses as indicator of participant's QoE, their frustration level measured continuously over the testing session using the Emotiv EPOC ${ }^{1}$ EEG system. Frustration was previously related to user's QoE with applications, for example relative to user's tolerance to delay [8].

\section{RELATED WORK}

\section{A. Multimedia QoE Assessment}

Various methods for assessing the multimedia quality, both subjectively by asking a group of subjects to rate the quality or objectively by computing metrics with the help of software programs [9], have been proposed.

A number of subjective methods for assessing the visual and audio-visual quality of multimedia applications were standardized in ITU-T Rec. P.910 [10] and ITU-T Rec. P.911 [11], which provide useful guidelines regarding the subjects and test material selection, the test environment setup, rating scales to be used for assessment, as well as methods for analysing the data. The main difference between the various methods consists in the way the test sequences are presented and rated such as: one-by-one presentation and rating of individual sequences (e.g., Absolute Category Rating method), rating of the difference between pairs of sequences (e.g., Degradation Category Rating method), or continuous quality rating over the entire sequence duration (e.g., Single Stimulus Continuous Quality Evaluation method).

The Mean Opinion Score (MOS) representing the average subjective quality ratings across all participants are usually represented on nominal scales, such as for example a 1 to 5 scale, where 1-Bad and 5-Excellent. Since one approach to interpret QoE is as the "overall acceptability of an application or service as perceived subjectively by the end-user" [12], mappings between MOS and the binary measure of acceptability have also been proposed [13].

\footnotetext{
${ }^{1}$ Emotiv, http://www.emotiv.com/
}

Subjective assessment of user perceived quality (QoP) was also extended to include user's satisfaction with the multimedia clip measured on a 6-level scale, together with his/her ability to perceive, synthesise and analyse the informational content, measured as the percentage of correct answers provided to a series of content-related questions [3].

Another mixed approach called Open Profiling of Quality (OPQ), which combines traditional analysis of user-provided quality ratings with quantitative data analysis, was also proposed [14]. By making use of various advanced statistical procedures for data analysis, OPQ was shown to provide a deeper understanding of user's QoE with 3D multimedia content by identifying important underlying factors related to content, viewing experience, as well as visual, audio and audiovisual quality (e.g., motion, clarity, colour, brightness, ease of viewing, eye strain, etc.) [15].

Objective video quality assessment metrics range from fullreference metrics that compare the quality of a clip relative to that of its reference unaffected by distortions, to reducedreference and no-reference metrics that require only some or no information regarding the original clip [16]. Existing objective metrics exploit various characteristics of natural images (e.g., edge, plane, texture, etc.), or of the human visual system (e.g., sensitivity to change in local gradient, contrast, blockiness, etc.) [17].

\section{B. Electroencephalography}

Electroencephalography (EEG) represents the process of recording electrical brain activity with the help of electrodes placed on the human scalp [18]. The recorded EEG signal is composed of multiple individual oscillations at different frequencies, which have been grouped in five major frequency bands or brain waves: delta, theta, alpha, beta, and gamma. While oscillations characteristic for all of these waves may be present in the overall EEG signal at the same time, one or another are usually dominant during certain states of awareness or different activities.

Various researchers have explored the possibility of using EEG as a means for detecting or differentiating between human basic emotions such as happiness, joy, distress, surprise, anger, fear, disgust and sadness [19], [20], [21], or between learning related emotions such as engagement, boredom and frustration [22], [23], [24].

In multimedia-related research areas, EEG has been used for automatic implicit emotional tagging of multimedia content, as an alternative to explicit approaches that require users to tag the clips themselves [25]. Furthermore, the possibility of using EEG for assessing user's perceived multimedia quality has also been explored. For example, the P300 EEG component consisting of a peak in the EEG signal voltage occurring approximately $300 \mathrm{~ms}$ after a significant event, was shown to have the potential of being used in order to assess whether users perceived changes in the multimedia quality [26], [27]. The authors showed that the more degraded a video is (i.e., due to compression), the earlier and higher the P300 amplitude is rising. 


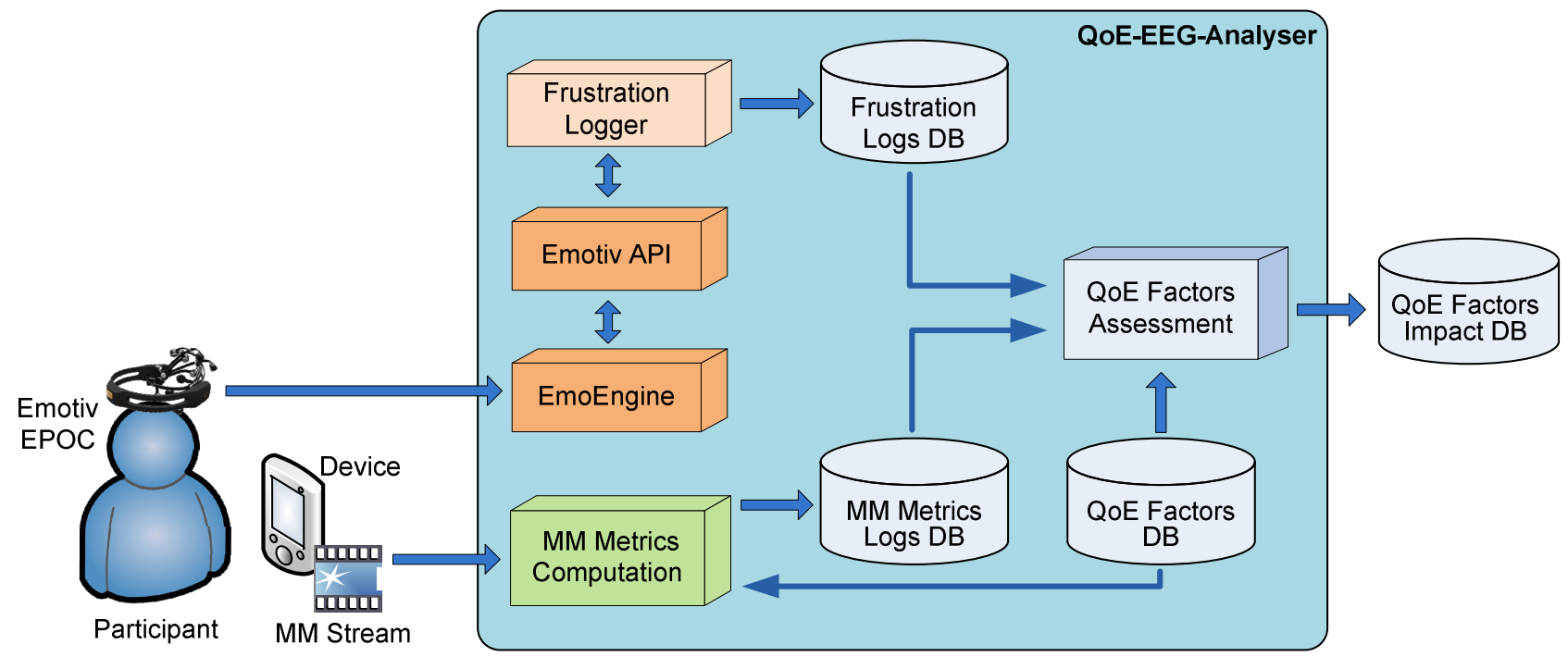

Fig.2. Block-level architecture of QoE-EEG-Analyser.

While traditional EEG equipments have a high cost and require a laborious setup that can take as much as one hour for a single participant [18], more recently a number of consumergrade EEG equipments (e.g., Emotiv EPOC, NeuroSky ${ }^{2}$ ), have started to be commercialised. Although these equipments present a number of limitations, such as lower number of sensors and functionalities, thanks to their affordable prices and reduced intrusiveness they draw the attention of an increasing number of researchers.

The Emotiv EPOC system in particular is a wireless brain computer interface device that records EEG signals from 14 electrodes. Along with raw EEG signals, the system enables to capture affective data (i.e., engagement, frustration, excitement and meditation), as well as expressive data (e.g., blink, right/left wink, look right/left, eyebrow raise, smile, laugh, etc.). Additionally, the device can be trained in order to enable brain controlled actions (e.g., moving objects) based on brain waves. The Emotiv EPOC was shown to provide accurate affective data when validated against questionnaires [28], [29].

Examples of research studies that have used the Emotiv EPOC system range from remote controlling objects such as a smartphone [30] or a robot arm [31], to measuring human emotional states during educational related studies [32], [33]. In multimedia-related research, the affective data provided by the Emotiv EPOC system was used in order to add context to user's tagging of multimedia clips [34].

Literature review shows that few research studies have explored the potential of using EEG for multimedia quality assessment. Furthermore, to the best of our knowledge no previous research used the affective data provided by the Emotiv EPOC system in order to enable automatic assessment and quantifying of individual factors on user's perceived multimedia quality.

\footnotetext{
${ }^{2}$ Neurosky, http://www.neurosky.com/
}

\section{QOE- EEG-ANALYSER}

\section{A. Block Architecture}

The block-level architecture of the proposed QoE-EEGAnalyser is presented in Figure 2. In order to assess and quantify the impact of various factors on user's QoE, a subjective study has to be conducted. During the study a number of participants are asked to view the test multimedia streams affected by various adaptive strategies or artifacts (e.g., variable resolution, bitrate, framerate, buffering, loss, etc.), named QoE factors thorough this paper.

While viewing the streams, participants' frustration level is continuously computed by the EmoEngine at a granularity of approximately one sample per second, based on the raw EEG data sent by the Emotiv EPOC headset. The frustration values measured on a continuous scale between 0 and 1 , are retrieved by the Frustration Logger through the Emotiv API, and saved to the Frustration Logs Database for further analysis.

In parallel, the MM (Multimedia) Metrics Computation component analyses the multimedia streams and computes a number of metrics that describe how the values of the corresponding QoE factors change over time. Metrics that are automatically extracted from the stream includes for example the video bitrate and resolution, if say the impact of varying these parameters with the available network bandwidth is analysed. The list of QoE factors under test (see Figure 1 for a list of possible factors), is taken from the QoE Factors Database, while the values of the metrics are stored in the $M M$ Metrics Logs Database.

Based on the information collected from multiple participants, the QoE Factors Assessment component assesses the impact of individual QoE factors on user's frustration. This is done by analysing how participants' frustration changes for various configurations of the $\mathrm{QoE}$ factor values occurring over the duration of the multimedia streams. The impact of a QoE factor is determined based on the relative frustration change it causes, and is stored in the QoE Factors Impact Database. 


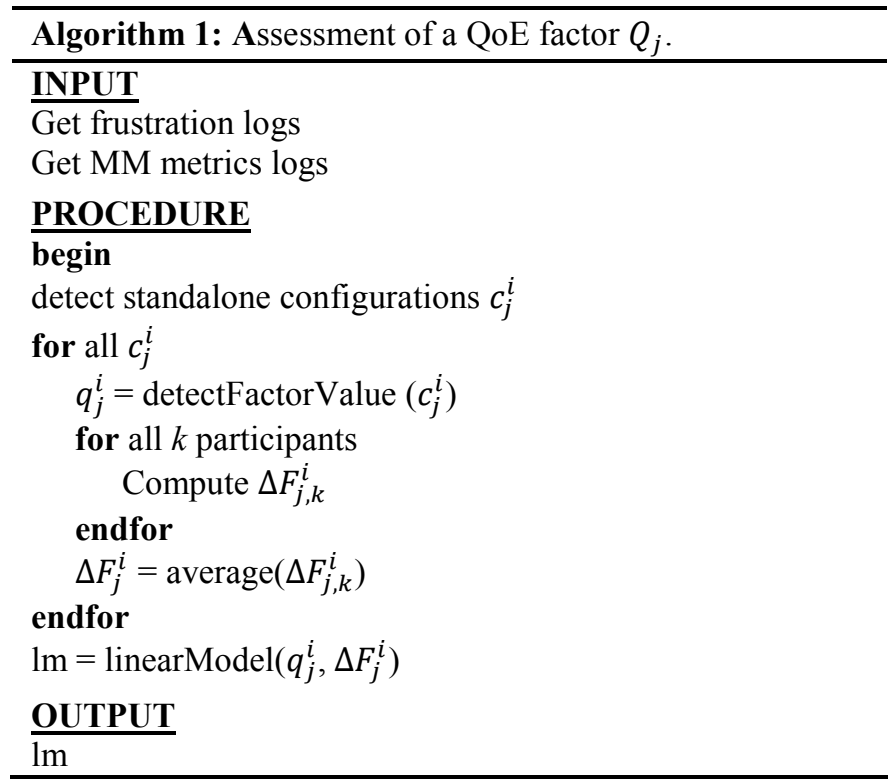

\section{B. Principle of QoE-EEG-Analyser}

As previously shown in Figure 1, there are various factors impacting user's QoE with multimedia services. Assume that there are $N$ factors whose impact has to be analysed, which are grouped in a set expressed as in (1).

$$
Q=\left(Q_{j}, j=\overline{1, N}\right)
$$

A specific QoE factor $Q_{j}$ can take different values $q_{j}^{i}$ on a continuous or discrete scale. For example, the QoE factor 'content type' can take a value in a predefined set (e.g., news, sports, cartoons, etc.), while the QoE factor 'video bitrate' can vary between a minimum and a maximum value for each measurement unit (e.g., frame, second, segment, clip, etc.).

Having the $\eta\left(Q_{j}\right)$ representing the number of independent values for a $Q_{j}$ factor, the total number of standalone configurations tested can be expressed as in (2). The standalone configurations $\left(c_{j}^{i}\right)$ corresponding to a QoE factor represent configurations when the value of that factor varies, while all the other factors are maintained constant.

$$
S=\sum_{j=1}^{N} \eta\left(Q_{j}\right)
$$

Since the absolute frustration value $(F)$ measured by the Emotiv EPOC system can vary between different participants for the same QoE factor configuration, QoE-EEG-Analyser uses the relative frustration change $\Delta F$, which is computed for a participant relative to his/her frustration level measured at the initial moment $\left(F_{r e f}\right)$, as in (3).

$$
\Delta F=\frac{F-F_{r e f}}{F_{\text {ref }}} \cdot 100[\%]
$$

The impact of a $\mathrm{QoE}$ factor $Q_{j}$, is determined as in Algorithm 1, based on the set of relative frustration changes $\Delta F_{j}^{i}$ corresponding to the $q_{j}^{i}$ independent values of the factor. Linear regression is used in order to assess and quantify the impact of the QoE factor.

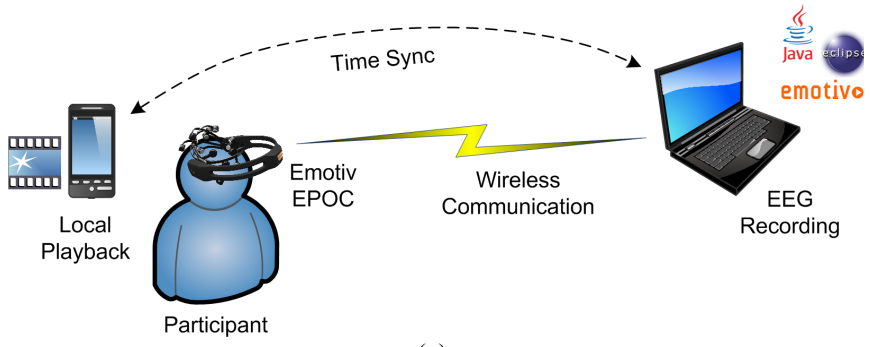

(a)

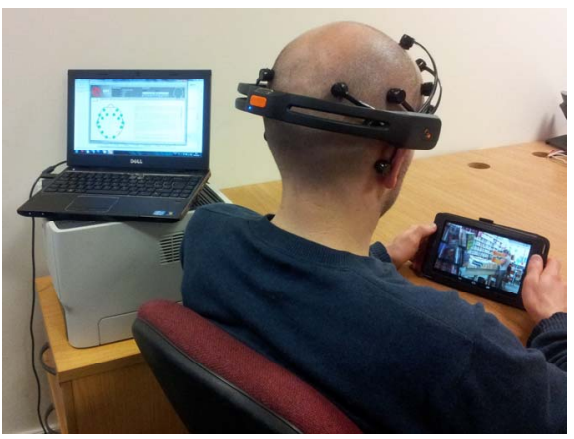

(b)

Fig.3. Subjective multimedia quality assessment study: (a) Graphical illustration of the test-bed setup; (b) Lab testing environment.

\section{SubJective CASE Study ANd Results}

A subjective study was conducted in order to analyse if the proposed QoE-EEG-Analyser, and in particular the approach of using the EEG-based frustration measures provided in realtime by the Emotiv EPOC system can be used in order to assess and quantify the impact of various factors on user's QoE. The study involved a number of participants watching and rating the quality of multimedia clips with changing quality levels, while wearing the Emotiv EPOC headset.

\section{A. Test Setup}

The evaluation test-bed is illustrated in Figure 3. A Google Nexus 7 tablet mobile device running on Android 4.2 operating system was used for displaying the multimedia clips used for testing. The device has a 7" LED-backlit IPS LCD capacitive touchscreen with a resolution of $1280 \times 800$ pixels. Furthermore it has an Nvidia Tegra $31.2 \mathrm{GHz}$ Quad-core ARM Cortex A9 CPU and 1 GB of RAM, being capable of playing full-HD $1080 \mathrm{p}$ videos without any buffering or playback issues.

A Dell Vostro 3350 laptop PC running Microsoft Windows 7 operating system was used to retrieve and save to log files the EEG data provided by the Emotiv EPOC headset, through the Emotiv API. The tablet device and the EEG recording PC were time synchronized in order to facilitate the data analysis.

\section{B. Multimedia Clips}

Four high-quality H.264 compressed educational multimedia clips with different content characteristics were used for the subjective study. The clips present both natural and non-natural (e.g., text) information, being representative 


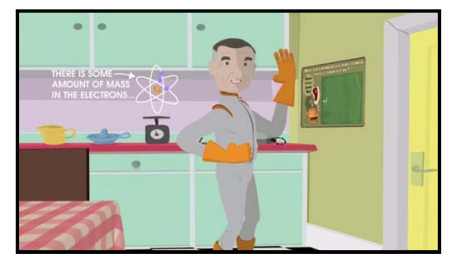

(a) AtomSize

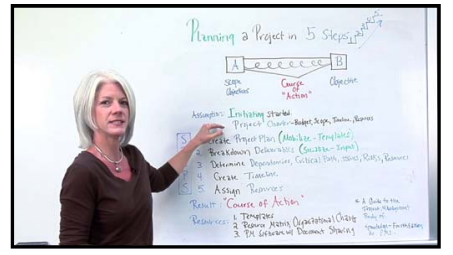

(c) ProjectPlanning

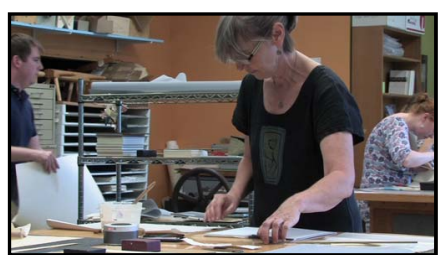

(b) ArtOfBook

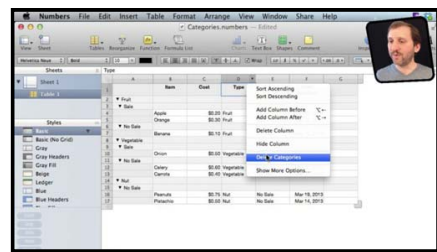

(d) CategoriesInNumbers

Fig. 4. Representative frames for the multimedia test clips.

TABLE I

Video ENCODING CHARACTERISTICS OF THE ORIGINAL MULTIMEDIA CliPS

\begin{tabular}{lllll}
\hline \multicolumn{1}{c}{\begin{tabular}{c} 
Multimedia \\
\multicolumn{1}{c}{ Clip }
\end{tabular}} & $\begin{array}{c}\text { H.264 Video } \\
\text { Compression } \\
\text { Profile }\end{array}$ & $\begin{array}{c}\text { Resolution } \\
\text { [pixels] }\end{array}$ & $\begin{array}{c}\text { Video } \\
\text { Bitrate } \\
\text { [kbps] }\end{array}$ & $\begin{array}{c}\text { Framerate } \\
\text { [fps] }\end{array}$ \\
\hline AtomSize & High@L4.0 & $1920 \times 1080$ & 2392 & 25 \\
ArtOfBook & Main@L3.1 & $1280 \times 720$ & 5061 & 29.97 \\
ProjectPlanning & High@L3.1 & $1280 \times 720$ & 1890 & 29.97 \\
CategoriesInNumbers & High@L3.1 & $1280 \times 720$ & 1843 & 29.97 \\
\hline \hline
\end{tabular}

for the broad category of educational multimedia clips. These were selected from large collections of educational clips that are freely available for download on iTunes $\mathrm{U}^{3}$ and Miro Guide $^{4}$. Figure 4 shows representative frames from each multimedia clip. A short description of the four clips is provided below.

- AtomSize - animation clip explaining the size of an atom; the clip was published by TEDEducation ${ }^{5}$.

- ArtOfBook - documentary, presenting the University of Iowa's Center for the Book program, available on University of Iowa's iTunes U page ${ }^{6}$.

- ProjectPlanning - presentation, explaining project management planning in five steps; the clip was produced by ProjectManager.com ${ }^{7}$.

- CategoriesInNumbers - screencast, explaining how to view the rows in spreadsheets by category using values in specific rows; the clip was produced by MacMost ${ }^{8}$.

Table I presents the video encoding characteristics of the 4 original multimedia clips. As already mentioned all of them were compressed using H.264/MPEG-4 AVC, at different

\footnotetext{
Apple iTunes U - Learn anything, anywhere, anytime, http://www.apple.com/ education/itunes-u/

${ }^{4}$ Miro Guide - Video Podcast Directory, http://www.miroguide.com/

Just How Small Is an Atom? - Jonathan Bergmann, http://ed.ted.com/lessons/just-how-small-is-an-atom\#watch

6 The Art of The Book, Iowa Magazine Segments, https://itunes.apple.com/ie/itunes-u/iowa-magazine-segments-hd/id413518586 5 Steps to Project Management Planning, http://www.projectmanager.com/5-steps-to-project-management-planning.php MacMost Now 845: Using Categories In Numbers, $\underline{\text { http://macmost.com/using-categories-in-numbers.html }}$
}

profiles. The AtomSize clip has a resolution of $1920 \times 1080$ pixels and a frame rate of 25 frames per second, while the rest of the clips have a resolution of $1280 \times 720$ pixels and a frame rate of 29.97 frames per second. The video bitrate of the test sequences varies between $1843 \mathrm{kbps}$ in case of the CategoriesInNumbers clip, and $5061 \mathrm{kbps}$ in case of the ArtOfBook.

The variation between source clips in terms of their encoding settings was unavoidable due to the unavailability of high-resolution and high-bitrate/ uncompressed educational clips of different content types, and also of sufficiently long duration to extract 5 minutes long test sequences required for the scenarios adopted in this study.

\section{Test Sequences and Scenarios}

The AtomSize clip was used for training the participants while ArtOfBook, ProjectPlanning and CategoriesInNumbers multimedia clips were used for the actual subjective study. One minute (60 seconds) long training sequence, and 5 minutes (300 seconds) long testing sequences were extracted from the original clips and used in the study.

Table II summarises the content characteristics of the three testing sequences in terms of their Spatial Index (SI), Temporal Index (TI) [10] and Colorfulness (CF) [35]. The three metrics are computed individually for each video frame, while their measure for the entire clip is obtained either by taking the maximum [10] or the average [36] across all frames.

TABLE II

TESTING SCENARIOS ENCODING SETTINGS

\begin{tabular}{lcccccccccc}
\hline \hline \multirow{1}{*}{$\begin{array}{c}\text { Test } \\
\text { Sequence }\end{array}$} & \multicolumn{4}{c}{$\boldsymbol{S I}$} & \multicolumn{4}{c}{$\boldsymbol{T I}$} & \multicolumn{4}{c}{$\boldsymbol{C F}$} \\
\cline { 2 - 11 } & Max & Avg & Std & Max & Avg & Std & Max & Avg & Std \\
\hline ArtOfBook & 46.4 & 12.5 & 6.5 & 81.9 & 2.5 & 3.7 & 73.7 & 33.1 & 12.0 \\
ProjectPlanning & 11.3 & 10.3 & 0.6 & 4.1 & 0.8 & 0.4 & 31.0 & 21.9 & 2.3 \\
CategoriesInNumbers & 13.1 & 10.6 & 0.7 & 6.9 & 0.1 & 0.3 & 20.1 & 14.0 & 1.2 \\
\hline \hline
\end{tabular}

Table III summarises the three testing scenarios that have been considered in the subjective study. The scenarios correspond to three different QoE factors: video bitrate, framerate and resolution. Six video bitrates were considered for Scenario 1, starting from $1800 \mathrm{kbps}$, and decreasing in steps of $300 \mathrm{kbps}$. The maximum bitrate and the bitrate step were selected based on the recommendation provided in [37],

TABLE III

TESTING SCENARIOS ENCODING SETTINGS

\begin{tabular}{|c|c|c|c|}
\hline $\begin{array}{c}\text { Testing Scenario } \\
\text { (QoE Factor } \\
\text { Analysed) }\end{array}$ & $\begin{array}{l}\text { Resolution } \\
\text { [pixels] }\end{array}$ & $\begin{array}{c}\text { Video Bitrate } \\
\text { [kbps] }\end{array}$ & $\begin{array}{c}\text { Framerate } \\
\text { [fps] }\end{array}$ \\
\hline $\begin{array}{l}\text { Scenario } 1 \\
\text { (Bitrate) }\end{array}$ & $1280 \times 720$ & $\begin{array}{l}1800,1500,1200, \\
900,600,300\end{array}$ & 30 \\
\hline $\begin{array}{l}\text { Scenario2 } \\
\text { (Framerate) }\end{array}$ & $1280 \times 720$ & 1800 & $\begin{array}{l}30,25,20, \\
15,10,5\end{array}$ \\
\hline $\begin{array}{l}\text { Scenario3 } \\
\text { (Resolution) }\end{array}$ & $\begin{array}{l}1280 \times 720 \\
854 \times 480 \\
640 \times 360 \\
426 \times 240 \\
320 \times 180\end{array}$ & $\begin{array}{l}\text { Constant compression } \\
\text { ratio } \sim 1.95 \mathrm{bits} / \mathrm{px} / \mathrm{sec} \\
\text { (i.e., } 1800,800,450,200 \text {, } \\
\text { 115) }\end{array}$ & 30 \\
\hline
\end{tabular}


of 1500-1800 kbps which are typical bitrates used for H.264 compressed video streaming at 720p resolution over Wi-Fi.

Six video framerates were considered for Scenario 2, ranging from $30 \mathrm{fps}$ to $5 \mathrm{fps}$, in steps of 5 frames per second. Five video resolutions were considered for Scenario 3, corresponding to the $720 \mathrm{p}, 480 \mathrm{p}, 360 \mathrm{p}, 240 \mathrm{p}$ and $180 \mathrm{p}$ common video profiles and the $16: 9$ aspect ratio. The video bitrates corresponding to the four smaller resolutions were selected so to maintain a constant compression ratio of 1.95 bits per pixel per second, as for the case of $1280 \times 720$ resolution at $1800 \mathrm{kbps}$.

A particular testing scenario consists of gradually decreasing the corresponding QoE factor value from the highest to the lowest value, going through all individual values. All three scenarios were run for each multimedia test sequence but in different order.

Long test sequences were selected in order to simulate a more natural viewing experience and to facilitate the analysis against the continuous measures provided by the Emotiv EPOC system. The 5 minutes length of the sequences enables to display each of the 17 test conditions across the three scenarios for 15 seconds.

In order to help the participants re-adjust their opinion for each scenario, an additional 15 seconds long segment at the highest quality (i.e., $1800 \mathrm{kbps}, 30 \mathrm{fps}, 1280 \times 720$ ), is displayed at the beginning of each testing scenario. However this segment are eliminated from the analysis as recommended for continuous quality assessment procedures in ITU-R Recommendation BT.500-12 [38].

AviSynth $^{9}$ was used for extracting the 15 seconds long segments from the original clips and for changing the video resolution and framerate accordingly. XMedia Recode ${ }^{10}$ media converter software, with $\times 264^{11}$ video encoder and FAAC ${ }^{12}$ audio encoder was used for compressing the tests segments in .mp4 containers. 2-pass average bitrate, Main profile, with CABAC and no B-frames, encoding settings for the H.264 codec were used for compressing the test sequences.

All videos presented audio component, which was maintained during the subjective testing for a more natural viewing experience, although the study has addressed only the video component. The audio quality was maintained constant across all test sequences and test cases (i.e., AAC-LC codec, $128 \mathrm{kbps}, 44.1 \mathrm{KHz}$ sampling rate).

\section{Methodology}

The Single Stimulus Continuous Quality Evaluation (SSCQE) [38] approach was used for video quality assessment during the multimedia playback. An Android application was developed for displaying the test sequences and quality rating. The quality rating was done on a continuous scale (i.e., 1 to 100), with the help of a vertical slider placed on the right hand side of the display and controlled by the participant through

\footnotetext{
${ }^{9}$ Avisynth - Main Page, http://avisynth.org/mediawiki/Main_Page

${ }^{10}$ XMedia Recode, http://www.xmedia-recode.de/

${ }^{11}$ VideoLAN-x264, http://www.videolan.org/developers/x264.html

12 AudioCoding - FAAC, http://www.audiocoding.com/faac.html
}

the touchscreen interface.

At the beginning of the testing session, each participant was explained the testing procedure, read information recommended by Emotiv about the EPOC system and EEG recording, and filled in a demographic questionnaire. The attendance was on a voluntary basis and verbal agreement on their attendance was obtained from each participant prior commencing the testing.

The testing session continued with setting up the Emotiv EPOC headset, while making sure that good signal is received from all sensors. After the training phase the actual testing was conducted by displaying the three test sequences ArtOfBook, ProjectPlanning and CategoriesInNumbers, in a random order for each participant.

Constant conditions were maintained for all participants. The multimedia test clips were played from the internal storage of the device in order to minimize any latencies and playback issues with buffering. The screen brightness was set at maximum value while the audio was played through the device speaker.

\section{E. Results and Analysis}

The performance of the proposed approach to use the frustration measure provided continuously by the Emotiv EPOC headset for video QuE assessment, was compared against the time sampled SSCQE subjective ratings provided through the on-screen slider by the participants while viewing the multimedia test sequences.

Figure 5 presents the distribution of participants based on their frustration change, as well as based on the change in their subjective video quality ratings, in case of the ArtOfBook test sequence. The results show that overall across the three testing scenarios the EEG frustration measure offers a better performance for detecting the decrease in video quality as opposed to the subjective video quality ratings. The frustration increased for $72.22 \%$ of the participants, and decreased for $27.78 \%$ of the participants. As opposed, only in case of $61 \%$ of the participants the video quality decrease occurring for the scenarios was confirmed by the SSCQE ratings, with the ratings increasing for $11.11 \%$ of the participants and remaining constant for $27.78 \%$ of the participants.

In case of Scenario 1 (video bitrate) the frustration increased for $66.66 \%$ of the participants and decreased for $33.33 \%$ of the participants, while the subjective ratings increased for $50 \%$ of the participants and remained constant for the other $50 \%$ of the participants.

In case of Scenario 2 (framerate) the frustration increased for $66.66 \%$ of the participants and decreased for $33.33 \%$ of the participants, while the subjective ratings increased for $66.66 \%$ of the participants, remained constant for $16.67 \%$ of the participants, and increased for $16.67 \%$ of the participants.

In case of Scenario 3 (resolution) the frustration increased for $83.33 \%$ of the participants and decreased for $16.67 \%$ of the participants, while the subjective ratings increased for $66.66 \%$ of the participants, remained constant for $16.67 \%$ of the participants, and increased for $16.67 \%$ of the participants.

These results also indicate that for this particular 


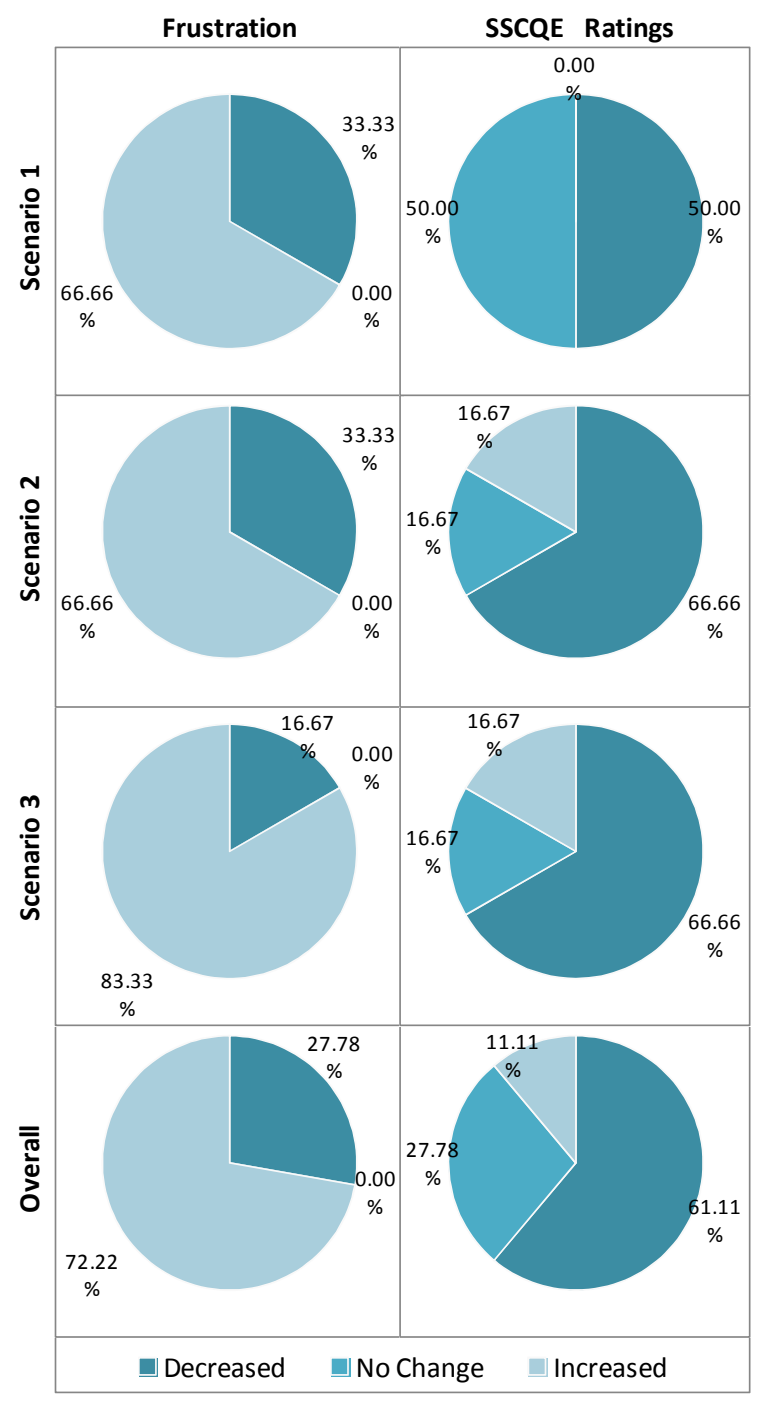

Fig.5. Distribution of participants based on their change in frustration and subjective SSCQE video quality ratings for the ArtOfBook test sequence.

multimedia clip the participants are more sensitive to a decrease in framerate and resolution as opposed to a decrease in video bitrate.

The proposed method offers the major advantage that it captures the impact of various $\mathrm{QoE}$ factors faster than traditional methods relying on user input.

Figure 6 presents the relative frustration change measures computed and averaged across all participants at a granularity of 1 second, over the duration of the three testing scenarios, for the ArtOfBook test sequence. The linear model is also illustrated in the figure.

While the relative frustration change measure presents oscillations in time, the results show that user's frustration tends to increase as the video quality decreases with the reduction in bitrate, framerate and resolution respectively. Estimated from the linear models, participants' frustration increased with $\sim 16 \%$ when gradually reducing the video bitrate from $1800 \mathrm{kbps}$ to $300 \mathrm{kbps}$, with $\sim 18 \%$ when gradually reducing the video framerate from $30 \mathrm{fps}$ to $5 \mathrm{fps}$, and with $\sim 42 \%$ when gradually reducing the video resolution
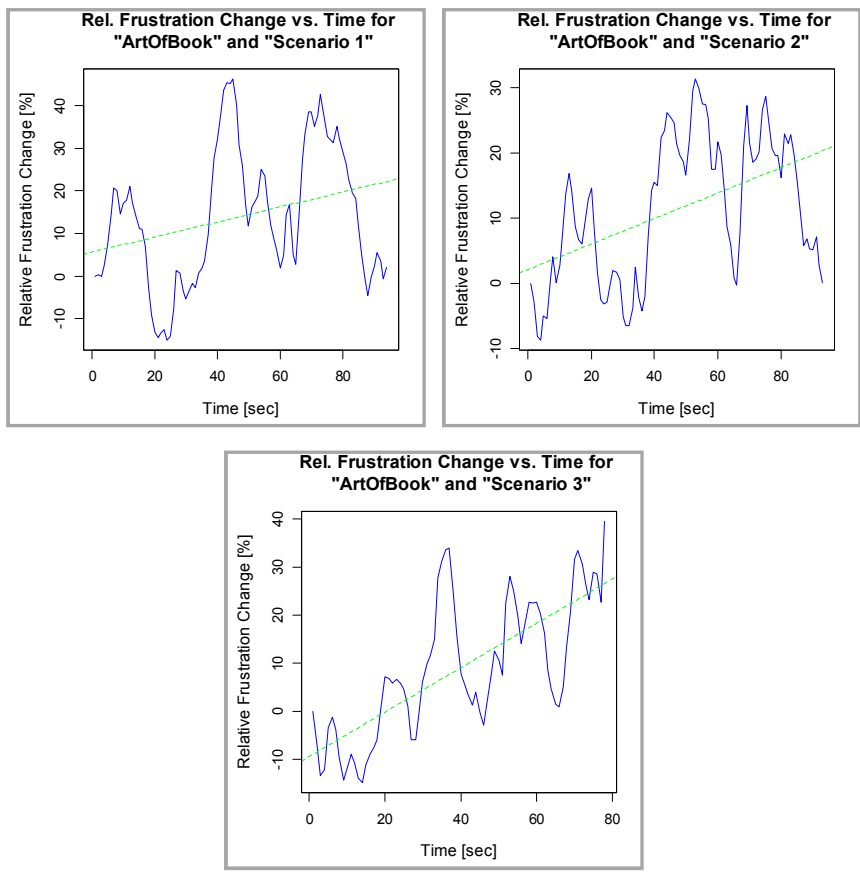

Fig.6. Relative frustration change measures computed every 1 second interval and averaged across all participants for ArtOfBook test sequence.

from $1280 \times 720$ to $320 \times 180$ (as well as the corresponding video bitrate from $1800 \mathrm{kbps}$ to $115 \mathrm{kbps}$ to maintain a constant compression ratio of $\sim 1.95 \mathrm{bits} / \mathrm{pixel} / \mathrm{second}$ ).

Therefore the results have shown that the proposed approach using the relative frustration change is feasible to be used in order to automatically assess and quantify the impact of various factors on user's multimedia QoE, without having to rely on subjective ratings provided directly by the user.

\section{CONCLUSION AND FUtURE WORK}

This paper proposed a user-centered solution for multimedia quality assessment based on EEG-based frustration measures provided in real-time by the Emotiv EPOC system. The proposed QoE-EEG-Analyser enables assessing and quantifying the impact of various factors on user's QoE in a non-invasive way, in the sense that participants do not have to provide input on their perceived experience. The proposed solution complements the existing subjective assessment methods, being particularly useful in situations when continuous assessment over the entire clip duration is necessary, such as for example assessing the impact of multimedia content adaptation strategies in real-like viewing scenarios. Future work is currently undertaken that extends the subjective analysis with higher number of clips, as well as more complex statistical analysis necessary to better assess and quantify the impact of different factors on user's QoE.

\section{REFERENCES}

[1] Cisco Systems, Inc., 'Cisco Visual Networking Index: Forecast and Methodology, 2011-2016', 30-May-2012. [Online]. Available: http://www.cisco.com/en/US/solutions/collateral/ns341/ns525/ns537/ns 705/ns827/white_paper_c11-481360.pdf. [Accessed: 15-Dec-2012]. 
[2] Google Inc., 'The New Multi-Screen World', Aug-2012. [Online]. Available: http://www.thinkwithgoogle.com/insights/featured/newmulti-screen-world-insight/. [Accessed: 15-Dec-2012].

[3] G. Ghinea and S. Y. Chen, 'Measuring quality of perception in distributed multimedia: Verbalizers vs. imagers', Computers in Human Behavior, vol. 24, no. 4, pp. 1317-1329, Jul. 2008.

[4] R. Trestian, A.-N. Moldovan, C. H. Muntean, O. Ormond, and G.-M. Muntean, 'Quality Utility modelling for multimedia applications for Android Mobile devices', in 7th IEEE International Symposium on Broadband Multimedia Systems and Broadcasting (BMSB 2012), Seoul, Korea, 2012, pp. $1-6$.

[5] B. Ciubotaru, G.-M. Muntean, and G. Ghinea, 'Objective Assessment of Region of Interest-Aware Adaptive Multimedia Streaming Quality', IEEE Trans. on Broadcast., vol. 55, no. 2, pp. 202-212, Jun. 2009.

[6] A.-N. Moldovan and C. H. Muntean, 'Subjective Assessment of BitDetect - A Mechanism for Energy-Aware Multimedia Content Adaptation', IEEE Transactions on Broadcasting, vol. 58, no. 3, pp. 480-492, 2012.

[7] A.-N. Moldovan and C. H. Muntean, 'Towards Personalised and Adaptive Multimedia in M-learning Systems', in Proceedings of 16th World Conference on E-Learning in Corporate, Government, Healthcare, and Higher Education (E-Learn 2011), Chesapeake, VA, USA, 2011, pp. 782-791.

[8] C. H. Muntean and J. McManis, 'End-User Quality of Experience Oriented Adaptive E-learning System', Journal of Digital Information, vol. 7 , no. 1,2006

[9] G.-M. Muntean, P. Perry, and L. Murphy, 'Objective and subjective evaluation of QOAS video streaming over broadband networks', IEEE Trans. Netw. Serv. Manage., vol. 2, no. 1, pp. 19-28, Nov. 2005.

[10] ITU-T, 'P.910: Subjective video quality assessment methods for multimedia applications', International Telecommunication Union, Geneva, Switzerland, Apr. 2008.

[11] ITU-T, 'P.911: Subjective audiovisual quality assessment methods for multimedia applications', International Telecommunication Union, Geneva, Switzerland, Dec. 1998.

[12] ITU-T, 'P.10/G.100 Amendment 2: New definitions for inclusion in Recommendation ITU-T P.10/G.100 - Vocabulary for performance and quality of service', International Telecommunication Union, Geneva, Switzerland, Jul. 2008.

[13] T. C. M. de Koning, P. Veldhoven, H. Knoche, and R. E. Kooij, 'Of MOS and men: bridging the gap between objective and subjective quality measurements in mobile TV', in Proceedings of Multimedia on Mobile Devices 2007, IS\&T/SPIE Symposium on Electronic Imaging, San Jose, CA, USA, 2007, vol. 6507, p. 11.

[14] D. Strohmeier, S. Jumisko-Pyykkö, and K. Kunze, 'Open Profiling of Quality: A Mixed Method Approach to Understanding Multimodal Quality Perception', Advances in Multimedia, vol. 2010, pp. 1-28, 2010.

[15] D. Strohmeier, S. Jumisko-Pyykkö, K. Kunze, and M. Bici, 'The Extended-OPQ Method for User-Centered Quality of Experience Evaluation: A Study for Mobile 3D Video Broadcasting over DVB-H', EURASIP Journal on Image and Video Processing, vol. 2011, no. 1, p. 538294, Feb. 2011.

[16] S. Winkler and P. Mohandas, 'The Evolution of Video Quality Measurement: From PSNR to Hybrid Metrics', IEEE Transactions on Broadcasting, vol. 54, no. 3, pp. 660-668, 2008.

[17] S. Chikkerur, V. Sundaram, M. Reisslein, and L. J. Karam, 'Objective Video Quality Assessment Methods: A Classification, Review, and Performance Comparison', IEEE Transactions on Broadcasting, vol. 57, no. 2, pp. 165-182, Jun. 2011.

[18] E. Harmon-Jones and D. M. Amodio, 'Electroencephalographic methods in psychology', H. Cooper, P. M. Camic, D. L. Long, A. T. Panter, D. Rindskopf, and K. J. Sher, Eds. Washington, DC, US: American Psychological Association, 2012, pp. 503-522.

[19] K. S. Park, H. Choi, K. J. Lee, J. Y. Lee, K. O. An, and E. J. Kim, 'Emotion recognition based on the asymmetric left and right activation', pp. $201-209,2011$.

[20] P. C. Petrantonakis and L. J. Hadjileontiadis, 'Emotion Recognition From EEG Using Higher Order Crossings’, pp. 186 -197, Mar. 2010.

[21] D. Nie, X.-W. Wang, L.-C. Shi, and B.-L. Lu, 'EEG-based emotion recognition during watching movies', presented at the 2011 5th International IEEE/EMBS Conference on Neural Engineering (NER), 2011, pp. $667-670$.

[22] A. Belle, R. Hobson, and K. Najarian, 'A physiological signal processing system for optimal engagement and attention detection', presented at the 2011 IEEE International Conference on Bioinformatics and Biomedicine Workshops (BIBMW), 2011, pp. 555-561.

[23] L. Shen, M. Wang, and R. Shen, 'Affective e-learning: Using "emotional" data to improve learning in pervasive learning environment', pp. 176-189, 2009.

[24] E. T. Mampusti, J. S. Ng, J. J. I. Quinto, G. L. Teng, M. T. C. Suarez, and R. S. Trogo, 'Measuring Academic Affective States of Students via Brainwave Signals', presented at the 2011 Third International Conference on Knowledge and Systems Engineering (KSE), 2011, pp. $226-231$.

[25] A. Yazdani, J.-S. Lee, and T. Ebrahimi, 'Implicit emotional tagging of multimedia using EEG signals and brain computer interface', in Proceedings of the first SIGMM workshop on Social media, New York, NY, USA, 2009, pp. 81-88.

[26] S. Scholler, S. Bosse, M. S. Treder, B. Blankertz, G. Curio, K.-R. Muller, and T. Wiegand, 'Toward a Direct Measure of Video Quality Perception Using EEG', IEEE Transactions on Image Processing, vol. 21, no. 5, pp. 2619-2629, May 2012.

[27] S. Arndt, J. Antons, R. Schleicher, S. Moller, and G. Curio, 'Perception of low-quality videos analyzed by means of electroencephalography', in 2012 Fourth International Workshop on Quality of Multimedia Experience (QoMEX), 2012, pp. $284-289$.

[28] R. Kuber and F. P. Wright, 'Augmenting the Instant Messaging Experience through the Use of BCI and Gestural Technologies', 2012.

[29] D. Cernea, A. Kerren, and A. Ebert, 'Detecting Insight and Emotion in Visualization Applications with a Commercial EEG Headset', 2011.

[30] M. Petersen, C. Stahlhut, A. Stopczynski, J. Larsen, and L. Hansen, 'Smartphones Get Emotional: Mind Reading Images and Reconstructing the Neural Sources', S. D'Mello, A. Graesser, B. Schuller, and J.-C. Martin, Eds. Springer Berlin / Heidelberg, 2011, pp. 578-587.

[31] G. N. Ranky and S. Adamovich, 'Analysis of a commercial EEG device for the control of a robot arm', presented at the Bioengineering Conference, Proceedings of the 2010 IEEE 36th Annual Northeast, 2010, pp. $1-2$

[32] J. Gonzalez-Sanchez, M. E. Chavez-Echeagaray, R. Atkinson, and W. Burleson, 'ABE: An Agent-Based Software Architecture for a Multimodal Emotion Recognition Framework', presented at the 2011 9th Working IEEE/IFIP Conference on Software Architecture (WICSA), 2011, pp. $187-193$.

[33] I. Ghergulescu, 'Automatic Non-Disturbing Motivation Monitoring in Game-based E-learning through Player Behaviour and EEG', Ph.D. Thesis, National College of Ireland, Dublin, Ireland, 2013.

[34] S. Davis, E. Cheng, I. Burnett, and C. Ritz, 'Multimedia user feedback based on augmenting user tags with EEG emotional states', in 2011 Third International Workshop on Quality of Multimedia Experience (QoMEX), 2011, pp. 143-148.

[35] D. Hasler and S. E. Suesstrunk, 'Measuring colorfulness in natural images', pp. 87-95, Jun. 2003.

[36] S. Winkler, 'Analysis of Public Image and Video Databases for Quality Assessment', IEEE Journal of Selected Topics in Signal Processing, vol. 6, no. 6, pp. 616-625, Oct. 2012.

[37] A. Molnar and C. H. Muntean, 'Cost-Oriented Adaptive Multimedia Delivery', IEEE Transactions on Broadcasting, vol. Early Access Online, 2013.

[38] ITU-R, 'BT.500-12: Methodology for the subjective assessment of the quality of television pictures', International Telecommunication Union, Geneva, Switzerland, Sep. 2009. 